\title{
Examination of tunnel junctions in the AIGaN/GaN system: Consequences of polarization charge
}

\author{
Madhusudan Singh ${ }^{\text {a) }}$ \\ Department of Electrical Engineering and Computer Science, University of Michigan, Ann Arbor, \\ Michigan 48109 \\ Yifei Zhang \\ Applied Physics Program, University of Michigan, Ann Arbor, Michigan 48109 \\ Jasprit Singh \\ Department of Electrical Engineering and Computer Science, University of Michigan, Ann Arbor, \\ Michigan 48109 \\ Umesh Mishra \\ Department of Electrical and Computer Engineering, University of California, Santa Barbara, \\ California 93106
}

(Received 25 May 2000; accepted for publication 24 July 2000)

\begin{abstract}
A strong piezoelectric effect and a large spontaneous polarization allow one to incorporate a large electric field $\left(>10^{6} \mathrm{~V} / \mathrm{cm}\right)$ and high sheet charge $\left(>10^{13} \mathrm{~cm}^{-2}\right)$ without doping in the AlGaN/GaN heterostructure. Theoretical studies are done to examine how polarization effects can be exploited to design metal-AlGaN/GaN tunnel junctions. We find that with a proper choice of $\mathrm{AlGaN}$ thickness undoped junctions can be made with very high metal to two-dimensional electron gas tunneling. Thus, a Schottky junction can be converted to a tunnel junction without doping. The tunneling probabilities approach those produced in a system doped at $\sim 4 \times 10^{19} \mathrm{~cm}^{-3}$. This work suggests that very interesting tunnel junctions can be made from undoped $\mathrm{AlGaN} / \mathrm{GaN}$ heterostructures. (C) 2000 American Institute of Physics. [S0003-6951(00)05138-X]
\end{abstract}

Group III-nitride semiconductors have attracted wide attention recently in view of their application in high power devices and optoelectronic devices with wavelengths ranging from the red into the ultraviolet. ${ }^{1-9}$

To understand the properties of the $\mathrm{AlGaN} / \mathrm{GaN}$ structure it is important to note that there is a spontaneous polarization present in the structures as a result of the cation and anion positions in the lattice. ${ }^{10}$ In heterostructures the difference between spontaneous polarization of two layers can be used to create a high density of mobile carriers. In addition to spontaneous polarization in heterostructures with strain (resulting from epitaxy) the piezoelectric effects for the nitride system are so large that effective built-in fields of $\sim 10^{6} \mathrm{~V} / \mathrm{cm}$ can be produced near the interfaces. ${ }^{11}$ This feature has been exploited to design $\mathrm{AlGaN} / \mathrm{GaN}$ high electron mobility transistors (HEMTs) with very high sheet charge without doping. It has been found experimentally ${ }^{12,13}$ that in nominally undoped HEMT structures sheet charge densities greater than $10^{13} \mathrm{~cm}^{-2}$ can be produced. Since the polarization charges effectively act as dopant charges, it is expected that with a proper understanding, tunnel junctions can be made from undoped metal-AlGaN/GaN structures. This letter sheds light on this issue.

Consider the case where the effective substrate is GaN and an $\mathrm{Al}_{x} \mathrm{Ga}_{1-x} \mathrm{~N}$ overlayer is grown coherently along the (0001) direction. The total polarization is found to have the value $^{12}$

a)Electronic mail: chhabra@eecs.umich.edu

$$
\begin{aligned}
P(x)= & P_{p z}+P_{s p} \\
= & \left(-3.2 \times x-1.9 x^{2}\right) \times 10^{-6} \mathrm{C} / \mathrm{cm}^{2} \\
& -5.2 \times 10^{-6} x \mathrm{C} / \mathrm{cm}^{2} .
\end{aligned}
$$

We see that in this system the effects arising from piezoelectric effect and spontaneous polarization mismatch are comparable. The electric field associated with the polarization given above is

$$
F(x)=\left(-9.5 x-2.1 x^{2}\right) \mathrm{MV} / \mathrm{cm} .
$$

We see that the built-in field and sheet charge values are very large. It is easy to produce fields around $10^{6} \mathrm{~V} / \mathrm{cm}$ and charge density around $10^{13} \mathrm{~cm}^{-2}$. In undoped $\mathrm{AlGaN} / \mathrm{GaN}$ field effect transistors such charge densities have been seen. ${ }^{13}$

To understand the tunneling problem we first need to use a charge control model to examine the band profile. Our charge control model first obtains the potential profile in a HFET structure by solving the Schrödinger equation and Poisson equation self-consistently.

The detailed formalism is described in Ref. 14. Once the band profile is known we use the WKB method to obtain the tunneling probability from the metal to the GaN channel.

In this letter we examine how the metal-semiconductor junction, shown in Fig. 1(a), behaves as far as tunneling of carriers is concerned. There is no doping in the structure and the two-dimensional charge is induced as a result of the fixed polarization charge at the $\mathrm{AlGaN} / \mathrm{GaN}$ interface. If we examine the density of the two-dimensional electron gas as a function of AlGaN barrier thickness, we see a behavior schemati- 

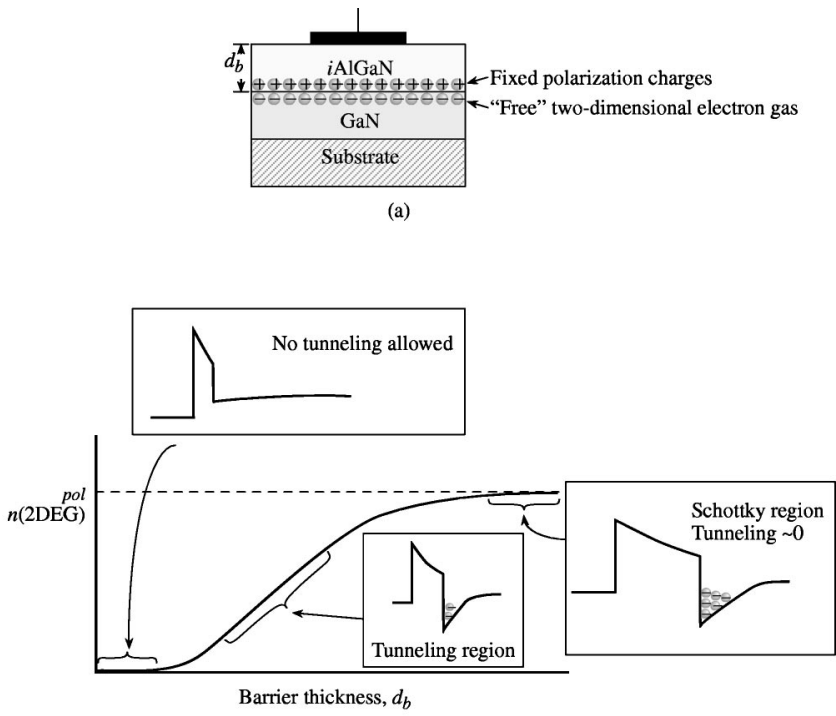

(b)

FIG. 1. A schematic of the metal-AlGaN/GaN structure examined. In the upper figure we show the fixed polarization charge and the induced twodimensional electron gas. In the lower part of the figure we show how a variation of the AlGaN thickness influences the two-dimensional electron gas and tunneling.

cally shown in Fig. 1(b). We see that there are three qualitatively different regions: (1) a region where the barrier region is very thick and the sheet charge is essentially equal to the polarization charge density (gate bias is zero). In this region there is no tunneling since the barrier for tunneling is quite large; (2) a region where the $\mathrm{AlGaN}$ thickness is very small and as a result the two-dimensional electron gas is not present. As the AlGaN thickness is decreased, the fixed polar charge at the AlGaN/GaN interface remains constant. However, the free carrier density it induces at the interface decreases due to the surface potential. In this case, as shown in Fig. 1 there are no allowed states to tunnel into from the metal and as a result there is no tunneling; (3) in the intermediate region the barrier thickness is large enough to allow two-dimensional electron gas (2DEG) and small enough to allow tunneling through the barrier. This is the region of interest for us in this letter.

In Fig. 2 we show the two-dimensional charge density and tunneling probability for electrons from the metal to the 2DEG region as a function of Schottky barrier height for four different $\mathrm{AlGaN}$ thicknesses. We have used a barrier with composition $\mathrm{Al}_{0.28} \mathrm{Ga}_{0.72} \mathrm{~N}$ for the studies presented here. It must be noted here that a higher Al content would achieve a higher interface charge density and require thinner $\mathrm{AlGaN}$ layers. However, there exists an upper limit on $\mathrm{Al}$ composition as the larger lattice mismatch makes pseudomorphic growth of $\mathrm{AlGaN}$ on GaN difficult, if not impossible. The Schottky barrier heights studied in this letter range from 0.2 to $1.4 \mathrm{~V}$. We see from Fig. 2 that as the barrier thickness decreases, the charge density in the $2 \mathrm{D}$ channel decreases. It also decreases as the Schottky barrier height increases.

As noted earlier, at too small a barrier thickness the $2 \mathrm{D}$ channel gets depleted and the energy levels in the heterostructure are above the energy levels near the metal Fermi level. To ensure that there are states in the semiconductor channel to tunnel into we carry out simulations for condi-
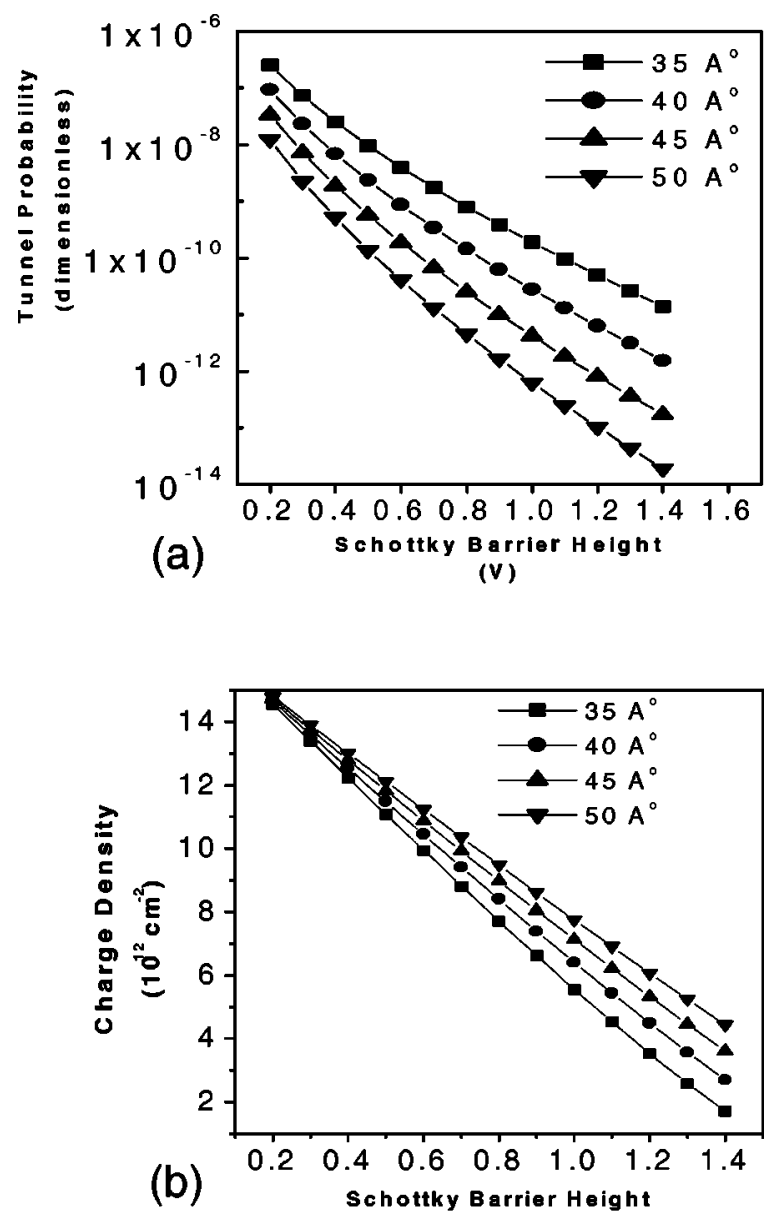

(v)

FIG. 2. Tunneling probability (a) and two-dimensional sheet charge density (b) as a function of the metal Schottky barrier height for four different of $\mathrm{AlGaN}$ barrier thicknesses. The Al mole fraction in the barrier is $28 \%$.

tions where the sheet charge in the channel is $\sim 10^{13} \mathrm{~cm}^{-2}$. For a fixed Schottky barrier height, this requires us to find the minimum thickness of the barrier at which $n_{2 D} \simeq 10^{13} \mathrm{~cm}^{-2}$. In Fig. 3 we show the results for this optimum thickness as a function of the barrier height. Also shown is the tunneling probability at this thickness. We see that the tunneling probability is $\sim 10^{-10}$ for a structure with a Schottky barrier height of $1.0 \mathrm{~V}$.

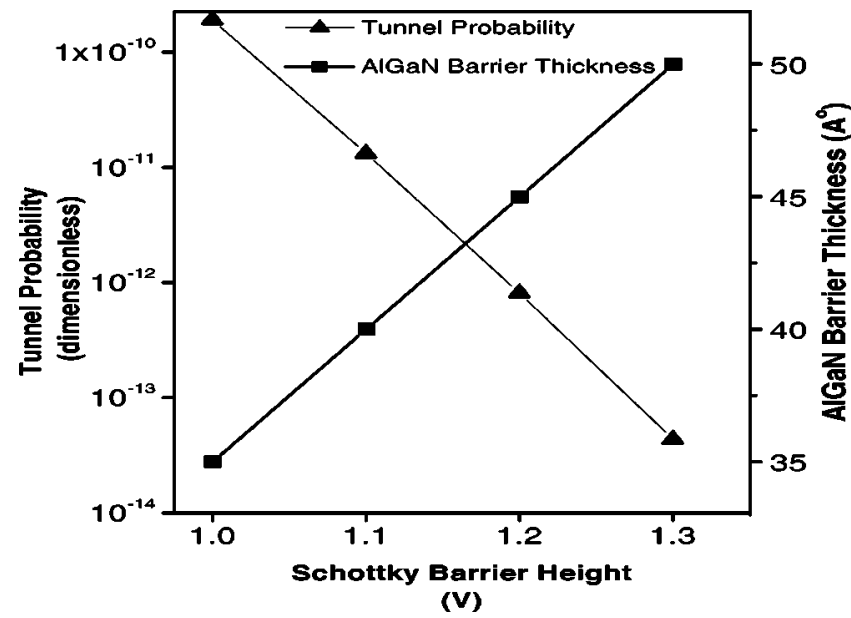

FIG. 3. Tunneling probability and optimized AlGaN barrier thickness as a function of the metal Schottky barrier height. 
It is well known that in semiconductor technology one way to convert a Schottky junction into an ohmic junction is to heavily dope the semiconductor. To illustrate the potential use of polarization effects, we consider the situation in which the AlGaN layer is absent and an attempt is made to form a conventional doping contact. We have carried out simulations to see what doping level would be needed to allow the same tunneling probability in this case (i.e., with a thick GaN barrier with a surface doping layer of $35 \AA$ with Schottky barrier $0.8 \mathrm{eV}$ ) as in the earlier structure (i.e., with a thin $\mathrm{AlGaN}$ layer with Schottky barrier $1.4 \mathrm{eV}$ ). The same thickness is chosen in both cases to remove any spurious effects arising from a difference in barrier thicknesses. The doping density needed is $\sim 4 \times 10^{19} \mathrm{~cm}^{-3}$. This represents an upper limit on doping of most semiconductors. Thus, using a polarization charge based contact bypasses an entire range of growth problems.

If one considers issues related to HEMT design, it is seen in light of this work that there exists a certain minimum thickness below which the polar charge induced 2DEG would be practically absent. This control of the 2DEG density in undoped GaN and ability to alter the nature of the metal-AlGaN/GaN junction from Schottky to Ohmic offers numerous benefits. For instance, the source and drain could be made on $\mathrm{AlGaN}$ region which have been etched down to these proper thickness while the gate could be deposited over a thicker region. Moreover, as indicated in Fig. 1(b), if the $\mathrm{AlGaN}$ thickness is very small, the junction reverts back to Schottky with no free mobile carriers. Such regions could be used for device isolation.

In summary, we have examined the tunneling behavior of metal-AlGaN/GaN junctions. We see that with proper structure optimization, it is possible to have junctions with high tunneling probability. This suggest that very interesting tunnel junctions acting as ohmic contacts or as nonlinear junctions can be designed using the built-in polarization charge at the $\mathrm{AlGaN} / \mathrm{GaN}$ interface.

This work has been supported by grants F001681 and F000629 from the US Office of Naval Research. The work at UC Santa Barbara has been supported by the ONR IMPACT program.

${ }^{1}$ I. Akasaki, H. Amano, S. Nagahama, T. Tanaka, and M. Kaike, Jpn. J. Appl. Phys., Part 2 34, L1517 (1995).

${ }^{2}$ S. Nakamura, M. Senoh, N. Iwasa, S. Nagahama, T. Yamada, and T. Mukai, Jpn. J. Appl. Phys., Part 2 34, L1332 (1995); S. Nakamura, M. Senoh, S. Nagahama, N. Iwasa, T. Yamada, T. Matsushita, Y. Sugimoto, and H. Kiyoku, Appl. Phys. Lett. 69, 3034 (1996).

${ }^{3}$ G. E. Bulman, K. Doverspike, S. T. Sheppard, T. W. Weeks, H. S. Kong, H. M. Dieringer, J. A. Edmond, J. D. Brown, J. T. Swindell, and J. F. Schetzena, Electron. Lett. 33, 1556 (1997).

${ }^{4}$ M. P. Mack, A. Abare, M. Aizcorbe, P. Kozodoy, S. Keller, U. K. Mishra, L. Coldren, and S. DenBaars, MRS Internet J. Nitride Semicond. Res. 2, 41 (1997).

${ }^{5}$ O. Aktas, Z. F. Fan, A. Botchkarev, S. N. Mohammad, M. Roth, T. Jenkins, L. Kehias, and H. Morkoc, IEEE Electron Device Lett. 18, 293 (1997).

${ }^{6}$ M. S. Shur and M. A. Kahn, MRS Bull. 22, 44 (1997).

${ }^{7}$ Y. F. Wu, S. Keller, P. Kozodoy, B. P. Keller, P. Parikh, D. Kapolnek, S. P. DenBaars, and U. K. Mishra, IEEE Electron Device Lett. 18, 290 (1997).

${ }^{8}$ U. K. Mishra, Y. F. Wu, B. P. Keller, S. Keller, and S. P. DenBaars, IEEE Trans. Microwave Theory Tech. 46, 756 (1998).

${ }^{9}$ R. Dimitrov, L. Wittmer, H. P. Felsl, A. Mitchell, O. Ambacher, and M. Stutzmann, Phys. Status Solidi A 168, R7 (1998).

${ }^{10}$ F. Bernardini, V. Fiorentini, and D. Vanderbilt, Phys. Rev. B 56, R10024 (1997).

${ }^{11}$ W. Q. Chen and S. K. Hark, J. Appl. Phys. 77, 5747 (1995).

${ }^{12}$ O. Ambacher, J. Smart, J. R. Shealy, N. G. Weimann, K. Chu, M. Murphy, W. J. Schaff, L. F. Eastman, R. Dimitrov, L. Wittmer, M. Stutzmann, W. Rieger, and J. Hilsenbeck, J. Appl. Phys. 85, 3222 (1999).

${ }^{13}$ I. P. Smorchkova, C. R. Elsass, J. P. Ibbetson, R. Ventury, B. Heying, P. Fini, E. Haus, S. P. DenBaars, J. S. Speck, and U. K. Mishra, J. Appl. Phys. 86, 4520 (1999).

${ }^{14}$ Y. Zhang and J. Singh, J. Appl. Phys. 85, 587 (1999). 